\title{
Hierarchical Zonation Technique to Extract Common Boundaries of a Layered Earth Model
}

\author{
Jayadev Acharya ${ }^{1}$, Goparaju Sreechakra ${ }^{1}$, Jaideva C. Goswami ${ }^{2}$, and Denis Heliot ${ }^{2}$ \\ ${ }^{1}$ Department of Electronics and Electrical Communication Engineering \\ Indian Institute of Technology, Kharagpur, WB 721 302, India. \\ ${ }^{2}$ Schlumberger Technology Corporation \\ 110 Schlumberger Dr., Sugar Land, TX 77478, U.S.A.
}

\section{INTRODUCTION}

Earth formations can be modeled as a layered medium with each layer having its own electrical, mechanical, and geometrical properties. In a typical exploration environment, a well-bore (about 15 to $30 \mathrm{~cm}$ in diameter) is drilled to a depth that may extend to a few kilometers. Formation properties are measured using multiple sensors moving along the well-bore trajectory (see figure 1a). To measure conductivity and permittivity, for instance, a sensor consisting of an array of transmitters and receivers is used (see Figure 1b). Transmitters are sequentially energized and phase and attenuation between receivers are measured. Measured phase and attenuations are then inverted for formation properties, namely layer-thickness, conductivity, dielectric constant, etc.

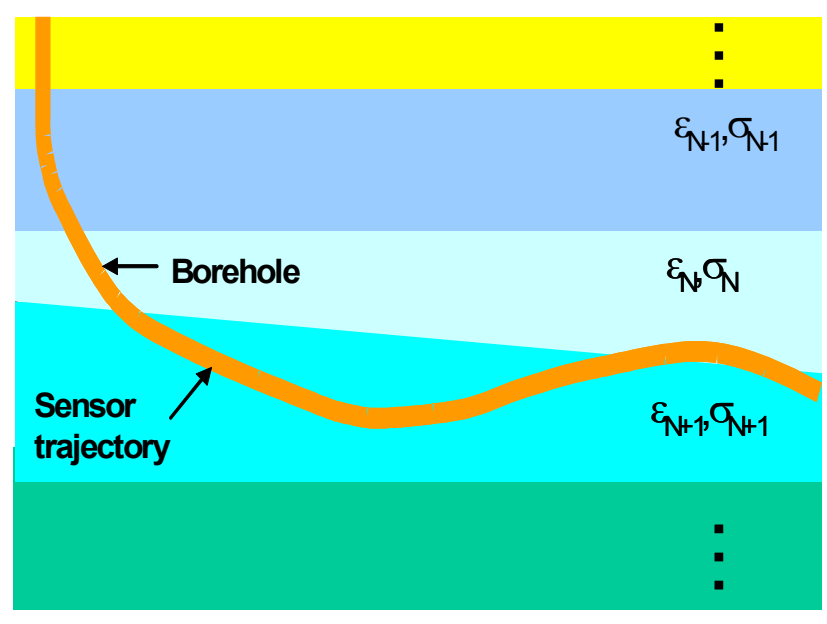

(a)

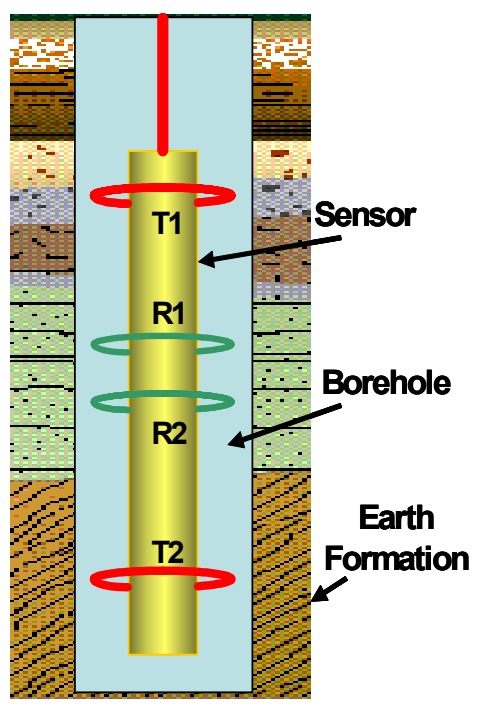

(b)

Figure 1: (a) Layers (beds) of earth formation and sensor trajectory. ( $\varepsilon$ : permittivity, $\sigma$ : conductivity), (b) a typical electromagnetic sensor in a borehole; T1 and $\mathrm{T} 2$ represent transmitting antennas and $\mathrm{R} 1$ and $\mathrm{R} 2$ are the receivers.

Data measured by the sensors are referred to as "log". In this paper, a hierarchical data segmentation method is presented to extract common boundaries from these logs. There are several methods for data segmentation. Recently a parametric classification technique [1] has been proposed to decompose data into multimodal components that provides the 
flexibility of choosing the underlying distribution. Algorithm [2] is based on optimization of variances. Here the goal is to minimize the within-class variance and to maximize between-class variance. This technique is computationally very inefficient. This deficiency has been tackled in [3-4].

Methods mentioned above deal with single log data. Often, we have multiple logs from same geological region measured by various sensors; each sensor having its own volume of investigation depending upon its resolution. In this work we describe a numerically efficient hierarchical segmentation method combining information from multiple sensors. The first step is preprocessing and normalization of logs and the second step is the classification that ensures minimum ratio of weighted within-class variance to weighted between-class variance at each level. To further improve the computational efficiency, we exploit the correlation among measured data. Such correlation helps reduce the dimensionality of segmentation problem by using principal component analysis (PCA). Hierarchical segmentation algorithm is then applied to principal component or a combination of a few major components.

The paper is organized as follows. In the next section we present the proposed algorithm. In Section III, we give numerical results. Finally, we conclude our work in Section IV.

\section{THEORY}

As mentioned in Section I, the data from multiple sensors are segmented hierarchically by minimizing the following objective functional representing the ratio of weighted within-class variance to weighted between-class variance:

$$
J(i)=\frac{\sum_{j=1}^{L} \alpha_{j} w_{j}(i)}{\sum_{j=1}^{L} \alpha_{j} b_{j}(i)}
$$

where $L$ is the number of logs corresponding to $L$ sensors and $w_{j}(i)$ and $b_{j}(i)$ denote the sampled within-class and sampled between-class variance [3] of the $j^{\text {th }}$ normalized log, and $\alpha_{j}$ are appropriate weights for each log. We assume that each sensor has the same sampling rate and acquires data in same geological region. Let $N$ be the number of data points in a log. Amplitude of $k^{\text {th }}$ segment after $i^{\text {th }}$ recursion is represented by $a_{k}(i)$, and $n_{k}(i)$ is the number of points in that segment. The number of segments, $C(i)$, after the $i^{\text {th }}$ recursion is one less than the previous recursion.

It can be shown that the within-class variance $w(i)$ and between-class variance $b(i)$ at the $i^{\text {th }}$ stage can be recursively computed as

$$
\begin{gathered}
w(i)=\frac{N-C(i-1)}{N-C(i)} w(i-1)+\frac{1}{N-C(i)} \times \frac{n_{p}(i-1) n_{p+1}(i-1)}{n_{p}(i-1)+n_{p+1}(i-1)}\left\{a_{p}(i-1)-a_{p+1}(i-1)\right\}^{2} \\
b(i)=\frac{C(i-1)-1}{C(i)-1} b(i-1)-\frac{1}{C(i)-1} \times \frac{n_{p}(i-1) n_{p+1}(i-1)}{n_{p}(i-1)+n_{p+1}(i-1)}\left\{a_{p}(i-1)-a_{p+1}(i-1)\right\}^{2}
\end{gathered}
$$

where $N$ is the total number of points in the log, $x_{k, m}(i)$ is the $m^{\text {th }}$ sample point in the $k^{\text {th }}$ segment after the $i^{\text {th }}$ recursion, and $a$ is the mean of the entire log. In the above expressions for variances we have dropped the subscript ' $j$ ' denoting each sensor. The same expression holds for each sensor. 
Denoting

$$
\xi_{p}^{2}(i)=\frac{n_{p}(i-1) n_{p+1}(i-1)}{n_{p}(i-1)+n_{p+1}(i-1)} \times\left\{a_{p}(i-1)-a_{p+1}(i-1)\right\}^{2}
$$

we observe that minimizing $\xi_{p}^{2}(i)$ minimizes $w(i)$ and maximizes $b(i)$ and hence optimizes the objective function. We find the minimum $\xi_{p}^{2}(i)$ value and then replace the amplitude of the corresponding two classes by their weighted mean. The number of points is sum of number of points of the merged classes. The new values of $\xi_{p}^{2}(i)$ for this new class with the adjoining classes can be easily calculated from the new amplitude. We see that we need to keep a track of only the parameter $\xi_{p}^{2}(i)$ and updating it using (4). The algorithm is repeated till we reach a desired number of classes. To further improve the computational efficiency, we apply principal component analysis to data and apply the above method to the principal component or a combination of major components.

\section{RESULTS AND DISCUSSION}

We first show the results for single log and then for multiple logs. Figure 2, illustrates log segments obtained from synthetic data. Originally, data had 16 segments. Next, we consider measured data using Schlumberger sensors at a depth of about 2.5 kilometers underground. We take three sensors data over a section of 120 meters. These sensors measure conductivity, density, and gamma ray radiation. It is clear from logs in Figure 3(a), that these sensors have different resolutions. Gamma ray sensor, for instance, has highest depth resolution. Using only one of these data to estimate bed boundaries may be erroneous. Common segments obtained by combining information from all three are shown in Figure 3(a).

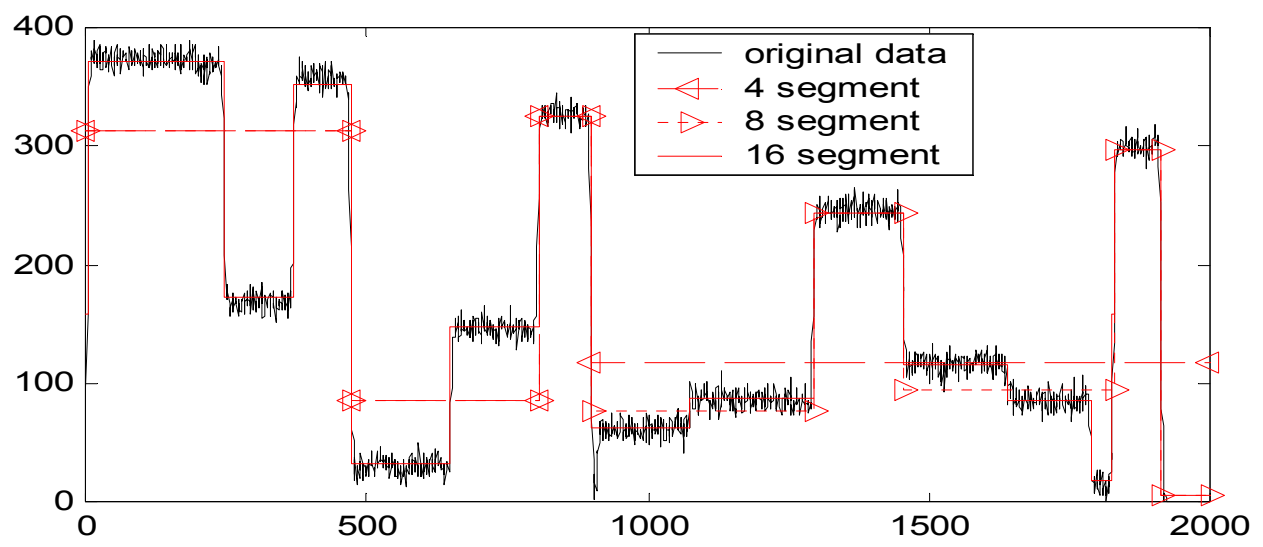

Figure 2: Segmentation of synthetic data.

Computation complexity increases linearly with the number of logs. We can reduce the effective number of logs (dimension) by exploiting the correlation among various measurements. As an example, we consider three logs of Figure 3(a), and perform PCA on these data; we obtain three components as shown in Figure 3(b). We have verified that by using only the first two of these components, we can get exactly same boundaries as Figure 3(a). In most cases, we have more than three measurements. By using only a few components of PCA we can reduce the time consumed and improve computational efficiency. 

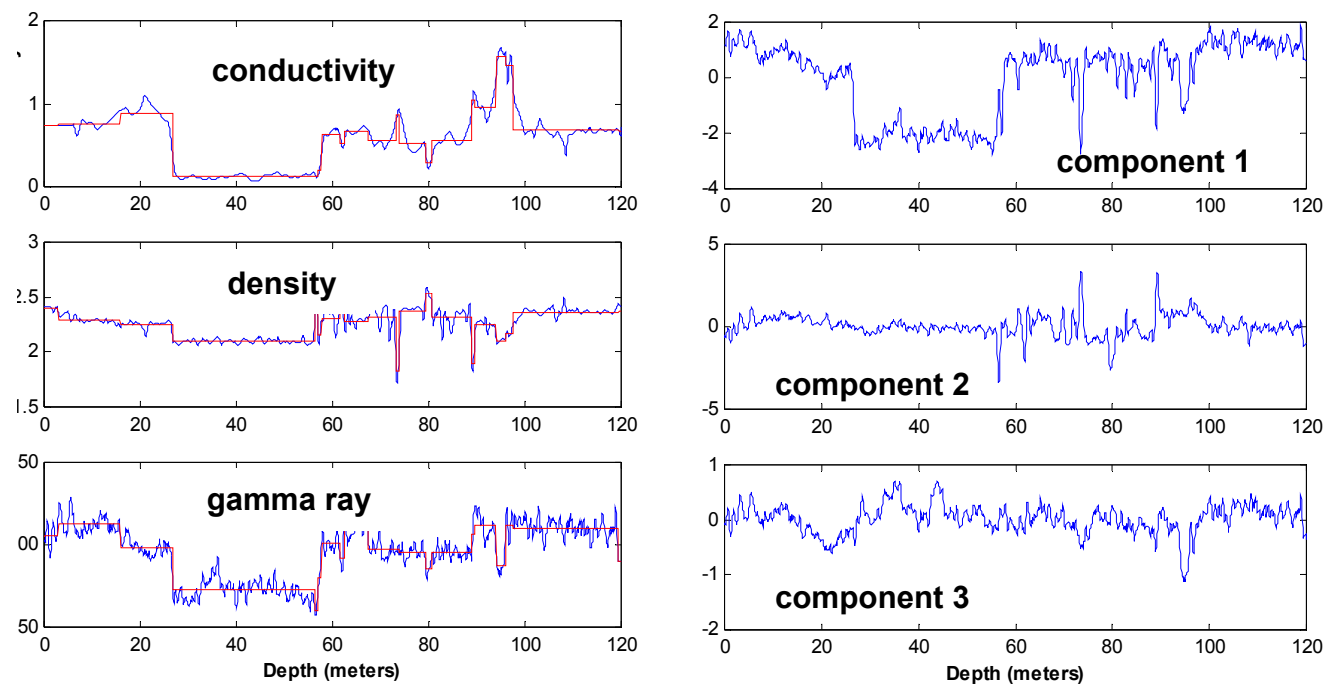

(a)

(b)

Figure 3: (a) Segmentation of multisensor data: conductivity $(\mathrm{S} / \mathrm{m})$, density $\left(\mathrm{gm} / \mathrm{cm}^{3}\right)$, gamma ray is in gAPI unit; a measure of radioactivity, (b) Various components obtained by applying PCA to data in (a)

\section{CONCLUSIONS}

In this work we have described a technique for segmentation of multiple logs to obtain common boundary. We have applied principal component analysis to further improve computation efficiency by exploiting inherent correlation among various measurements. As a future work, we plan to develop statistical approach for automatic termination of the algorithm; that is, to have a priori estimation of number of final segments.

\section{REFERENCES}

[1] J. C. Goswami, A. Srivastava, D. Heliot, T. J. Neville, "Method for transmitting wellbore data acquired in the wellbore to the surface", U.S. Patent 7,133,777, Nov. 2006.

[2] J. D. Testerman, "A statistical and reservoir-zonation technique," J. Petrol. Tech., vol. 14, pp. 889-893, 1962.

[3] A. Moghaddamjoo, "Constraint Optimum Well-Log Signal Segmentation," IEEE Tran. on Geo. and Remote Sensing, Vol. 27, No.5, pp. 633 - 641, Sept. 1989.

[4] R. L. Kirlin and A. Moghaddamjoo, "A robust running-window detector and estimator for step-signals in contaminated Gaussian noise," IEEE Trans. Acoust., Speech., Signal Processing, vol. 34, no. 4, pp. 816-823, 1986. 